\title{
Frequency dependence of microwave complex permeability under magnetic bias
}

\author{
Artem O. Shiryaev*, Stanislav Y. Bobrovskii, Aleksey V. Osipov, Dmitry A. Petrov, Konstantin N. Rozanov, and \\ Sergey N. Starostenko
}

ITAE RAS, 125412, Moscow, Izhorskaya 13, Russian Federation

\begin{abstract}
Measurement of the frequency dependence of the permeability under magnetic bias is proposed as a new method for studying the microwave magnetic properties of magnetic materials. The samples under study are either rolls of thin ferromagnetic films or the composites filled with sendust particles. It is shown that the permeability measured under external magnetic bias depends on sample thickness. The correct interpretation of the measured data is possible only when sample demagnetization is accounted for. The values of the anisotropy field and the saturation magnetization of thin nitrogen doped iron films and flakeshaped sendust particles are calculated considering demagnetization of the washer-shaped sample.
\end{abstract}

\section{Introduction}

For the characterization of microwave properties of magnetic materials, ferromagnetic resonance (FMR) measurements with fixed frequency and swept magnetic bias is frequently used [1-3]. This technique is usually performed in a high-quality cavity and has significantly higher signal-to-noise ratio, but the calculated permeability data may differ from that ones obtained under zero bias and the technique is poorly suited for measurements within a range of frequencies. Also, the measurement frequency is typically fixed and not very high, about $10 \mathrm{GHz}$. The FMR frequency of the measured sample may be above $10 \mathrm{GHz}$, or FMR may be observed at a low permanent magnetic field and the sample may not be saturated. However, the measurement technique allows easy variation of sample shape, size and orientation.

This technique allows finding the anisotropy field and the saturation magnetization using Kittel's equation:

$$
f_{\text {res }}=\gamma \sqrt{\left(H_{k}+H_{\text {bias }}\right)\left(H_{k}+H_{\text {bias }}+4 \pi M_{0}\right)} \text {, }
$$

where $f_{\text {res }}$ is a FMR frequency, $H_{\text {bias }}$ is the external magnetic bias, at which FMR is observed, $H_{k}$ is the anisotropy field, $4 \pi M_{0}$ is the saturation magnetization, and $\gamma$ is the gyromagnetic ratio. Equation (1) is valid for elongated particles and thin ferromagnetic films [4].

An alternative way of microwave magnetic measurement may be a measurement of frequency dependence of complex permeability $\mu$ under external magnetic bias [5]. The permeability is conventionally measured in a coaxial line with high accuracy in a wide frequency range up to $20 \mathrm{GHz}$. The calibration technique for the coaxial line is well elaborated. However, samples of the coaxial form are nonuniformly magnetized, in contrast to samples of ellipsoidal shape. The response of such samples to the external bias is described by a sum of depolarization factors [6]. Often an effective depolarization factor, which depends not only on the shape and orientation of the sample, but also on its permeability, is used [7]. The problem of demagnetization of a coaxial washer is well studied in the application to the problem of creating a coilgun $[8,9]$.

The demagnetization factor for ring with rectangular cross-section in the direction along axis is described with the equation:

$$
N_{z}(\sigma, \tau)=\frac{1}{\tau\left(1-\sigma^{2}\right)} \int_{0}^{+\infty} \frac{d q}{q^{2}}\left(1-e^{-2 q \tau}\right)\left[J_{1}(q)-\sigma J_{1}(\sigma q)\right]^{2},
$$

where $\sigma$ is the ratio of the inner radius to the outer radius, $\tau$ is the ratio of the thickness to the outer diameter, and $J_{l}$ is the Bessel function.

Because of the sample magnetization, it is necessary to use a field inside the sample, weakened by demagnetization, instead of the external magnetic bias. This field depends on the depolarization factor $N$ and the static permeability $\mu_{s t}$ of the sample:

$H_{i}=H_{\text {bias }}\left(1+4 \pi\left(\mu_{s t}-1\right) N\right)$.

Previously this method without demagnetization account was used only for qualitative estimations of high-frequency properties [4, 10-13]. Accounting for demagnetization allows the magnetic characteristics of the samples to be found quantitatively. Also a microstrip line is often used for measurements under bias [14], but the frequency range is not wide, up to $8 \mathrm{GHz}$.

The research is aimed at studying the demagnetization effect on the measured permeability of coaxial washer-shaped samples. 


\section{Experiment}

Two types of coaxial samples are under study: rolls made of thin flexible iron films and composites with paraffin wax as host matrix filled with sendust (Fe-SiAl) particles.

Iron films of submicrometer thickness are deposited by the RF magnetron sputtering with an iron target onto a flexible mylar substrate [15]. The deposition is performed in $\mathrm{Ar}+\mathrm{N}_{2}$ atmosphere at the room temperature and the pressure of $10^{-4}$ Torr. The fraction of $\mathrm{N}_{2}$ in the sputtering chamber is $10 \%$. Due to the nitrogen admixture, the iron of the deposited films contains dissolved nitrogen and is further referred to as Fe-N. The thickness of the film is $0.27 \mu \mathrm{m}$.

The composite samples are prepared by mixing flake sendust powder with molten paraffin. The mixture is pressed in a shape of $2 \mathrm{~mm}$ thick washers fit for a standard $7 / 3 \mathrm{~mm}$ coaxial line.

The frequency dependencies of microwave permittivity and permeability of the samples are measured by the Nicolson-Ross-Weir technique in $7 / 3$ coaxial line in the frequency range of 0.1 to $20 \mathrm{GHz}$. The coaxial cell is placed in a coil producing magnetic bias up to 930 Oe directed along coaxial axis.

\section{Thin ferromagnetic films}

Because of sample demagnetization, rolls of different thicknesses behave differently under magnetic bias. The measured data is shown in Fig. 1. The black lines correspond to the $5.4 \mathrm{~mm}$ roll thickness, the gray lines to $2.5 \mathrm{~mm}$. The strength of the external permanent magnetic bias is shown in the figure. With magnetic bias increase, the static permeability decreases and the FMR frequency shifts toward higher frequencies. The measured permittivity does not depend on bias. Under 190 Oe external bias, the difference between permeabilities of the rolls of different thickness is maximal. The further bias increase leads to the difference decrease due to drop of static permeability.

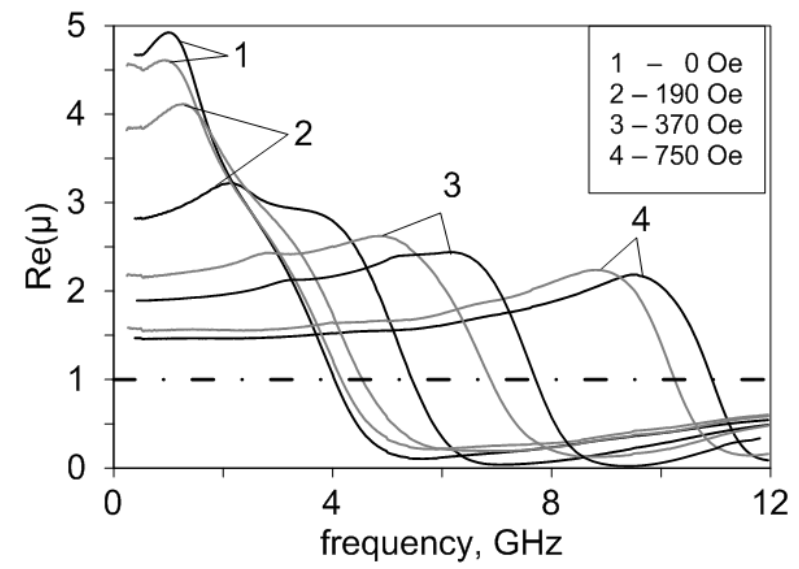

Fig. 1. The measured frequency dependence of the real permeability of coaxial rolls made of thin Fe-N films under magnetic bias (black lines correspond to $5.4 \mathrm{~mm}$ sample thickness, gray lines correspond to $2.5 \mathrm{~mm}$, the numbers show the magnetic bias).
The dependence of the FMR frequency on the strength of magnetic bias is shown in Fig. 2. The circles correspond to the dependency of the FMR frequency on the strength of external bias, and the triangles correspond to dependency on the field inside the sample, with the account for demagnetization. The demagnetization factors in the direction along coaxial axis are taken from $[8,9]$. For the coaxial sample of $7 \mathrm{~mm}$ external diameter, $3 \mathrm{~mm}$ internal diameter and $2.5 \mathrm{~mm}$ thickness, the demagnetization factor is 0.6 . For the $5.4 \mathrm{~mm}$ sample thickness the demagnetization factor is 0.35 . The FMR frequency dependence on the internal field is shown by the same single curve, which can be approximated by Eq. (1). The measurement at 0 Oe doesn't fit the curve, because the sample under zero bias is unsaturated.

The approximation (1) makes it possible to find the saturation magnetization and the anisotropy field values. The obtained values of the anisotropy field is $\mathrm{H}_{\mathrm{k}}=57$ Oe and the saturation magnetization is $\mathrm{M}_{0}=1480 \mathrm{G}$. The reference book value of saturation magnetization for iron is $1700 \mathrm{G}$. The difference may be related to the nitrogen doping of the sputtered film.

Vectors of the strength of the permanent external magnetic bias and microwave magnetic field are perpendicular to each other and are parallel to the film plane. The correct interpretation of the measured data is possible for isotropic films only.

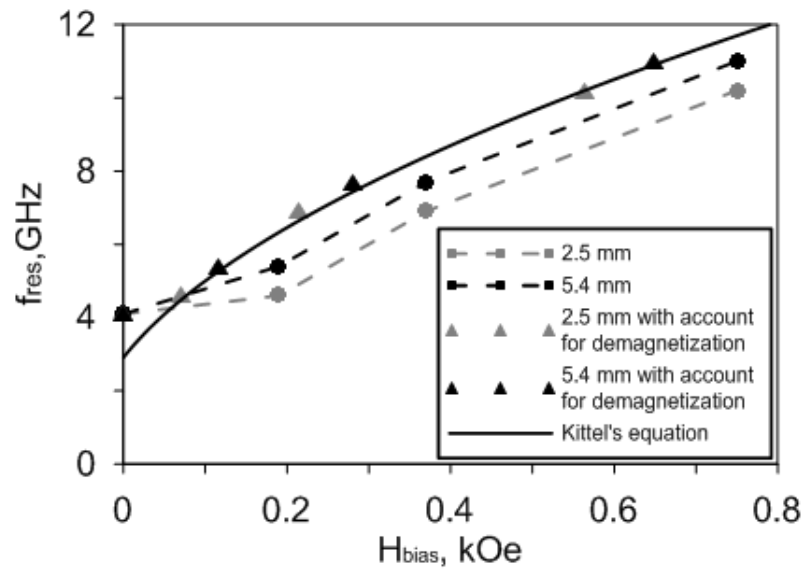

Fig. 2. The measured dependence of the FMR frequency on the strength of magnetic bias: the circles correspond to the measured data without accounting the sample demagnetization, the triangles correspond to the measured data with accounting the sample demagnetization, the black curve corresponds to the approximation by Kittel's equation.

\section{Flake-filled composites}

The measured frequency dependencies of the permeability of composites filled with different fractions of the sendust particles is shown in Fig. 3. The volume fraction of the inclusions in the samples under study is 2 , 4, 7.4, 11.6 and $14.4 \%$.

In the absence of the external magnetic bias, the FMR frequency for all fillings is close to $3 \mathrm{GHz}$. The effective properties of composites containing inclusions with low demagnetization factors typically obey the Wiener mixing rule [16]. 

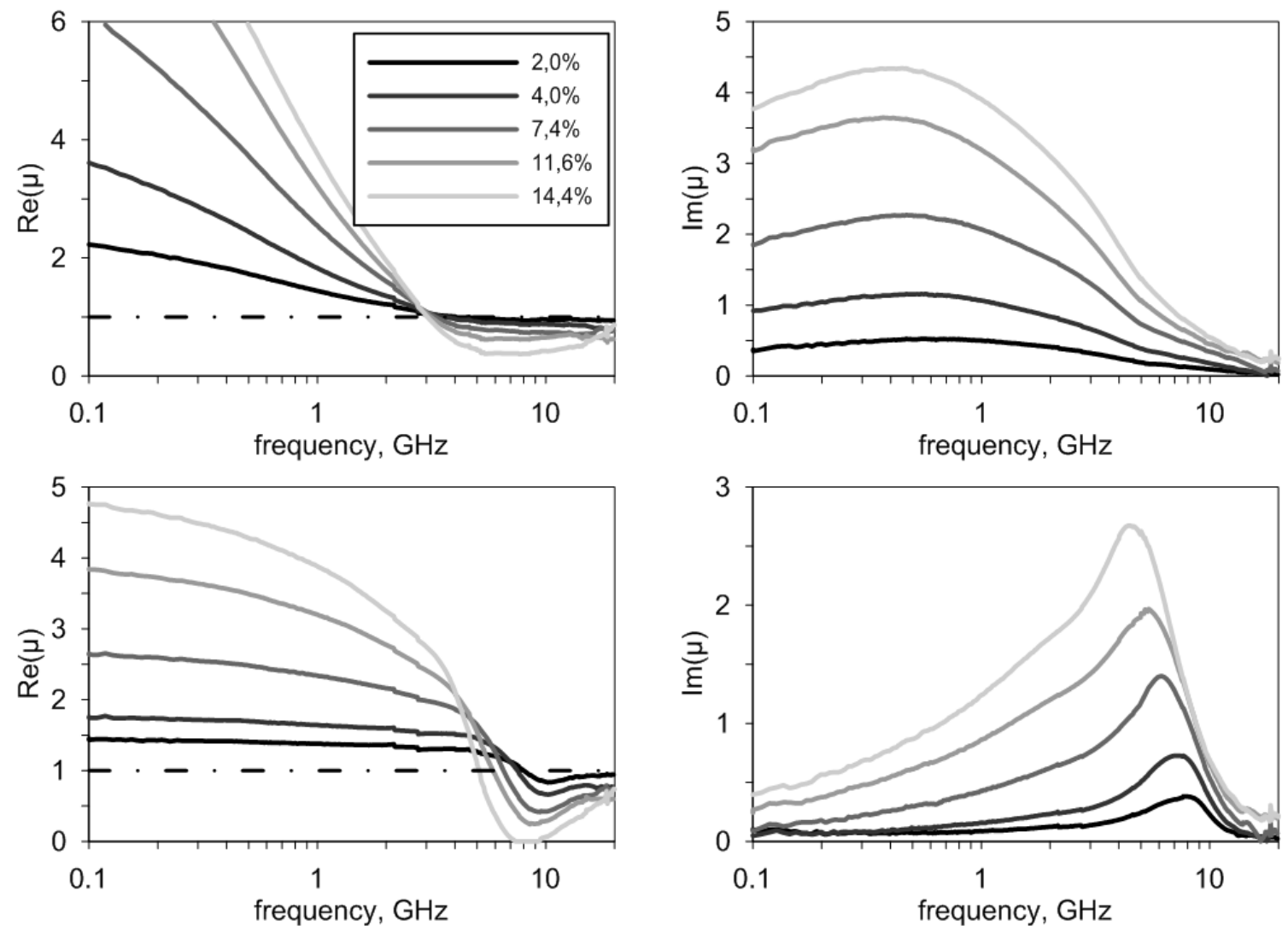

Fig. 3. The frequency dependence of the complex permeability at different volume concentrations of inclusions in composite material: no external magnetic bias (top), external magnetic bias of $930 \mathrm{Oe}$ (bottom), lines of different colors denote different volume fraction of inclusions.
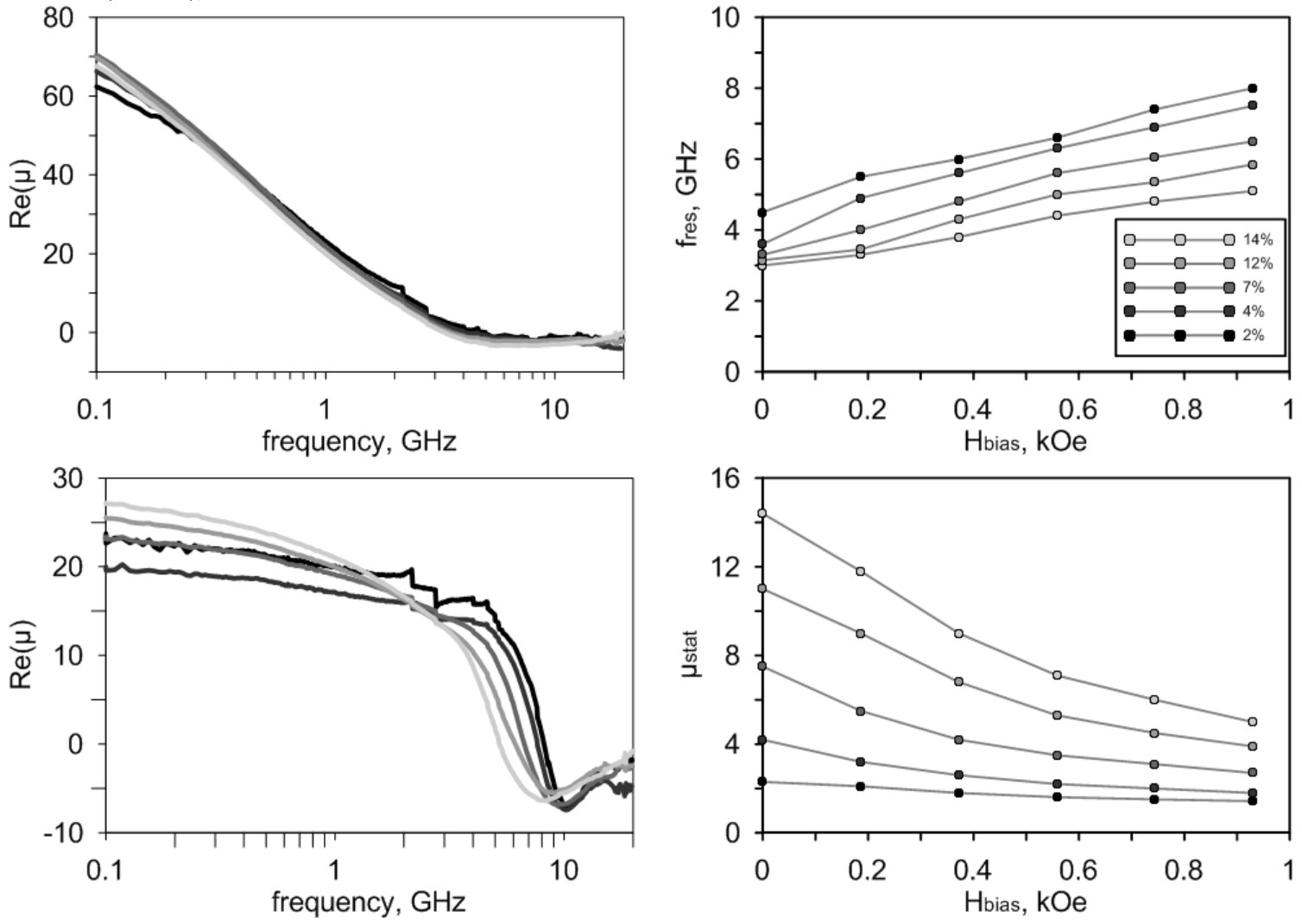

Fig. 4. The frequency dependence of the real intrinsic permeability under 0 magnetic bias (top) and under 930 Oe magnetic bias (bottom), lines of different colors denote different volume fraction of inclusions.

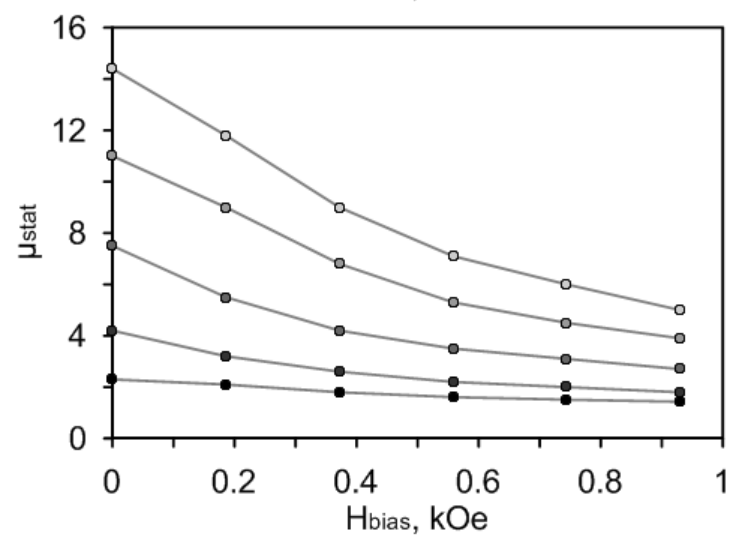

Fig. 5. The measured dependencies of the FMR frequency (top) and static permeability (bottom) of the composites on the external magnetic bias, darker circles denote smaller volume fraction of inclusions. 
Therefore the intrinsic susceptibility of sendust particles may be obtained as the ratio of composite susceptibility to the volume concentration. The frequency dependence of intrinsic permeability of sendust particles is shown in Fig. 4. Under zero bias the intrinsic permeability is the same for all fillings.

However, an increase of the external magnetic bias leads to a shift in the resonant frequencies, and the more is the concentration of the sendust in the composites, the larger is the static permeability of the composite and the less is the FMR frequencies shift. Therefore the intrinsic permeability under bias depends on filling, which is inexplicable within the framework of known theories.

Dependencies of the FMR frequency and static permeability of the composites on the strength of the external bias for different fillings are shown in Fig. 5.

The dependence of the composite FMR frequency on bias is reduced to a single curve, when sample demagnetization is accounted for by Eq. (3). The values of the anisotropy field and the saturation magnetization of the sendust particles are found by fitting the parameters of Kittel's equation (1), the fitted results are shown in Fig. 6. The fitted parameters correspond to $H_{k}=160$ Oe and $M_{0}=645 \mathrm{G}$. The handbook value of saturation magnetization for sendust is $780 \mathrm{G}$.

In case of the composite samples the data scatter is higher than in case of the rolled films. Possible reasons are the contribution of flake orientation in the composite, difference of shape of FMR curve from the Lorentzian type, and the inaccuracy of static permeability determination.

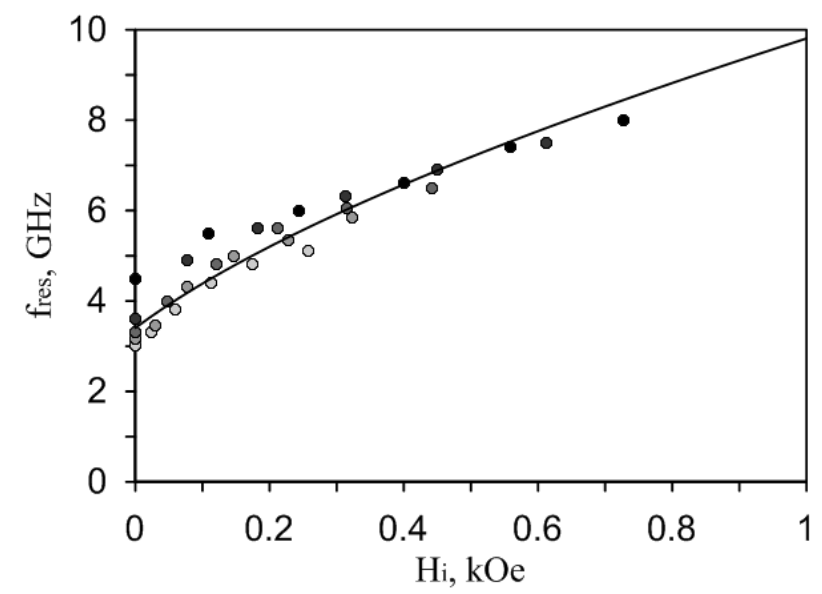

Fig. 6. The measured dependencies of the FMR frequency on the strength of the magnetic bias inside the sample: the circles correspond to the measured data with account for the sample demagnetization, the black curve corresponds to the approximation by Kittel's equation.

\section{Conclusion}

Measurement of the frequency dependence of the permeability under magnetic bias is proposed as a new method for studying the microwave magnetic properties of magnetic materials. The samples under study are either rolls of thin ferromagnetic films or the composites filled with sendust particles. It is shown that the permeability measured under external magnetic bias depends on sample thickness. The correct interpretation of the measured data is possible only when sample demagnetization is accounted for. The values of the anisotropy field and the saturation magnetization of thin nitrogen doped iron films and flake-shaped sendust particles are calculated considering demagnetization of the washer-shaped sample.

\section{Acknowledgement}

The study has been supported by Russian Foundation for Basic Research (RFBR); Project no. 18-08-00491.

\section{References}

1. R.M. Bozorth, Ferromagnetism (IEEE Press, New York, 1993)

2. I. Neudecker, G. Woltersdorf, B. Heinrich, T. Okuno, G. Gubbiotti, C.H. Back, J. Magn. Magn. Mater. 307, 148 (2006)

3. S.S. Kalarickal, P. Krivosic, M. Wu, C.E. Patton, M.L. Shnider, P. Kabos, T.J. Silva, J.P. Nibarger, J. Appl. Phys. 99, 093909 (2006)

4.

5. I.T. Iakubov, O.Yu. Kashurkin, A.N. Lagarkov, S.A. Maklakov, A.V. Osipov, K.N. Rozanov, I.A. Ryzhikov, S. N. Starostenko, J. Magn. Magn. Mater. 324, 3385-3388 (2012)

6. S.N. Starostenko and K.N. Rozanov, PIER C 8, 43 (2009)

7. D.J. Bergman, Phys. Rev. B. 19, 2359 (1979)

8. D.J. Bergman, K.J. Dunn, Phys. Rev. B. 45, 13262 (1992)

9. M. Beleggia, D. Vokoun, M. De Graef, J. Magn. Magn. Mater. 321, 1306 (2009)

10. C.P. Bidinosti, Y. Sakamoto, K. Asahi, IEEE Magn. Lett. 5 (2014)

11. I. T. Iakubov, A. N. Lagarkov, S. A. Maklakov, A. V. Osipov, K. N. Rozanov, I. A. Ryzhikov, V. V. Samsonova, and A. O. Sboychakov, J. Magn. Magn. Mater. 321, 726 (2009).

12. M. Yamaguchi, S. Yabukami, K.I. Arai, Sensors and Actuators 81, 212-215 (2000)

13. L. Liu, K.N. Rozanov, M. Abshinova, Appl Phys A 110, 275-279 (2013)

14. K. Chalapat, J. Timonen, M. Huuppola, L. Koponen, C. Johans, R. Ras, O. Ikkala, M. Oksanen, E. Seppala, and G. Paraoanu, Nanotechnology 25, 485707 (2014)

15. C. Brosseau, S. Mallégol, P. Quéffelec, and J. Youssef, Phys. Rev. B 70, 092401 (2004)

16. I.T. Iakubov, A.N. Lagarkov, S.A. Maklakov, A.V. Osipov, K.N. Rozanov, I.A. Ryzhikov, S.N. Starostenko, J. Magn. Magn. Mater. 272-276, 2208 (2004)

17. K.N. Rozanov and M.Y. Koledintseva, J. Appl. Phys 119, 073901 (2016) 